\title{
Prácticas corporales y conversión al oficio entre tatuadores y perforadores
}

\section{Melania Stehli (UNR/CONICET/ \\ $U N L)$}

INTRODUCCIÓN

Nuestro trabajo articula procesos de consumo cultural vinculados a prácticas corporales, en relación con dinámicas de profesionalización de oficios no convencionales consolidados durante la década del 90 en algunos países de América del Sur, como el de tatuador y perforador.

El germen se inicia por un interés personal por conocer algunas de las transformaciones vinculadas al cuerpo en las sociedades contemporáneas. Para ello, y a priori, seleccionamos un grupo social que consideramos nos iba a permitir observar esas cuestiones, los tatuadores y perforadores.

Cuando comenzamos a realizar el trabajo de campo, fueron emergiendo dimensiones, procesos, agentes y trayectorias, que se nucleaban de forma densa alrededor de las prácticas y espacios que circundan al tatuaje y la perforación, reformulando nuestro interés inicial. Así, reconocimos dinámicas de construcción y negociación de significados que subsidiaban procesos de construcción identitarios, en diálogo conflictivo con los contextos subjetivos y sociales que enmarcaban la trayectoria social y profesional de los actores.

En su trabajo sobre los significados del tatuaje en mujeres de clase media de Porto Alegre, Leitão reconoce que la historia social del tatuaje y la perforación en occidente (aunque no asimilables) está caracterizada por una existencia como otredad, es decir, como lo exótico, marginal o portador de una moralidad negativa (Leitão 2003: 5).

Asociado primeramente a los criminales y las prostitutas, como también a los marineros y a los espectáculos circenses, es recién en la década del 50 que en algunos lugares de Estados Unidos comienza a darse una transformación de la clientela que frecuenta los locales, estás prácticas pasan a ser repropiadas y resignificadas por diversos grupos en las décadas subsiguientes que - en términos 
generales -, buscaban sintetizar símbolos de transgresión y manifestaciones contraculturales, en disconformidad a la cultura mayoritaria en donde se expresaba (Leitão 2003: 8).

Aunque actualmente estas prácticas se encuentren mundializadas, ello no implica que en cada configuración urbana, económica y cultural, se desarrollen con temporalidades similares o significados unívocos. Así, desarrollar la "biografía social" de estas modificaciones corporales (Kopytoff 1991) en el contexto específico de la ciudad de Santa Fe adquiere relevancia, porque los trabajos empíricos disponibles se han realizado sobre configuraciones socioculturales dispares, como es el caso algunas de las ciudades capitales de América del Sur (San Pablo, Santiago de Chile, Buenos Aires o Rio de Janeiro) ${ }^{1}$.

Ello permite indagar el impacto sociocultural que posee en algunos contextos, y en nuestro caso, la función que desempeña en la construcción de biografías e identidades, al establecer un contrapunto entre procesos y configuraciones simbólicas vinculadas a estos oficios -autoclasificaciones, formas de aprendizaje y procesos de profesionalización- y, por otro lado, las características que adquieren sus reapropiaciones en contextos locales. Como bien resume Kopytoff,

(...) la biografía memorable de una cosa se convierte en la historia de las diversas singularizaciones experimentadas por ella de las clasificaciones y reclasificaciones a las cuales ha estado sujeta en un mundo incierto de categorías cuya importancia varía con cada modificación menor del contexto (Kopytoff 1991: 119).

Ahora bien, el referente empírico son los discursos y las prácticas relativas al oficio de algunos tatuadores y perforadores profesionales ${ }^{2}$ que ejercen el oficio y ofertan sus servicios en locales comerciales ubicados en la ciudad de Santa Fe, Argentina.

Como veremos, los oficios analizados conservan un formato de reproducción tradicional, pero algunas de las características de sus ejecutantes, de sus productos y de las relaciones sociales que los atraviesan, evidencian fenómenos y transformaciones sociales que podemos documentar desde los análisis y conceptualizaciones de los sociólogos de la modernidad, como las visiones acerca de la globalización, los procesos de destradicionalización e individualización actuales, entre otros (Beck et al. 1997).

El trabajo de campo lo realizamos sobre los locales comerciales como espacios de observación porque es donde los tatuadores y perforadores ejecutan y comercializan su servicio (las prácticas de modificación corporal) de manera oficial y pública, a los que el consumidor no tiene el acceso restringido o vedado y en tanto la concentración, afluencia y heterogeneidad de clientes es mayor ${ }^{3}$ (Costa 2004: 26).

El acercamiento hacia los locales de la zona céntrica y comercial de la ciudad se funda en la necesidad de conocer los significados, prácticas y el entramado de relaciones, desde la voz de los actores, en el momento de la interacción con los consumidores y durante la ejecución de las modificaciones corporales, porque interesa la forma en que el mundo es comprendido, experimentado, producido por los sujetos (Vasilachis de Gialdino 2006:29).

Durante el ingreso preliminar al campo nos encontramos con una escasez de información tanto nacional como local, como también con la ausencia de registro sobre el número de ejecutantes de estos oficios o de datos estadísticos acerca del consumo de estas prácticas, que contrastaba con la información disponible, tanto científica como periodística, acerca de estas prácticas en otros países, como Brasil o Chile. 
Por lo mencionado, nuestro interés es indagar el trabajo de tatuador y perforador, sus características, sus lógicas internas y la existencia de sistemas clasificatorios comunes, como condicionante central de las prácticas de modificación corporal y las representaciones sobre el cuerpo de los actores.

En lo que sigue nos ocuparemos de tres ejes. En primer lugar, analizaremos el tipo de ingreso y la forma de aprendizaje del oficio; luego, reconstruiremos las etapas del proceso de conversión al oficio y sus elementos constitutivos, haciendo hincapié en los estándares de profesionalización internos a cada subgrupo y algunas de las implicancias de la comercialización; por último, buscaremos comprender qué piensan los tatuadores y perforadores acerca de las prácticas de modificación corporal que realizan a otros y a sí mismos.

Veremos luego que la autorrealización de tatuajes y perforaciones por parte de los oficiantes configura un factor de distanciamiento de los valores y las formas de vida del entorno familiar y social. En los relatos, estas formas de transgresión social con el cuerpo como centro son coronadas con la“conversión"4 al oficio de tatuador y perforador.

Estos procesos subjetivos dan la pauta de la vigencia y relevancia de la destradicionalización contemporánea para comprender los distanciamientos de las tradiciones heredadas, y asimismo, de la impronta del proceso de individualización como recurso para analizar los modos actuales de construir caminos personales y la necesidad de hacerle frente a la responsabilidad de los actos (Beck y Beck-Gernsheim 2003).

Por ende, las similitudes en torno a los procesos de consolidación de los oficios analizados permite apuntalar hipótesis y hallazgos de trabajos en configuraciones sociales dispares (Braz 2005; Costa 2004; Ferreira 2006; Leitão 2003). Así, se abre la potencialidad de conocer los contextos a través de los usos y efectos de prácticas expresivas, como establece Ferreira, "as diferentes modalidades de mobilização do corpo por parte destes tornarse-ão assim nos próprios instrumentos de uma leitura das condições sociais da sua produção e representação" (Ferreira 2006:20).

Por la naturaleza del objeto construido y de los objetivos propuestos elegimos una estrategia cualitativa. El carácter descriptivo del trabajo y su acento en el mundo de vida fundamentan el uso de la estrategia de recolección y análisis de datos propuesta, privilegiando su especificidad interpretativa, inductiva, multimetódica y reflexiva, centrada en la práctica real, situada, de los sujetos (Vasilachis de Gialdino 2006: 29) 5 .

\section{FORMAS DE INGRESO EN EL OFICIO Y GENERACIONES DE OFICIANTES: AUTODIDACTAS Y APRENDICES}

Como sabemos, los tatuajes son una modificación del color de la piel en el que se crea un dibujo y se plasma con agujas estériles que inyectan tinta o algún otro pigmento bajo la epidermis de una persona, y los tatuadores son sujetos que realizan modificaciones corporales, diseños o dibujos, en el cuerpo de las personas y a ellos mismos.

Los perforadores (piercing) realizan la práctica de perforar una parte del cuerpo humano, generalmente para insertar aros de formas y dimensiones variadas. Además estas perforaciones, encontramos nuevas modificaciones corporales como los branding, expansores y escarificaciones (incluso suspensiones corporales), denominadas extremas o - como establece Braz en base en su trabajo etnográfico- "poco convencionales" (2005: 170)6. 
Ambas prácticas son una forma de modificación corporal (o alteración del cuerpo) que supone el cambio permanente o semipermanente deliberado del cuerpo humano por motivos no médicos.

Estas formas prácticas de vivir e interactuar con el cuerpo, suponen diferentes conocimientos prácticos sobre el mismo, que se complementan con saberes teóricos o técnicos constitutivos de cada oficio. Estos saberes son, en el caso de los tatuadores, los conocimientos artísticos en complemento con el aprendizaje de la técnica de tatuar sobre la piel y, en el caso de los perforadores, los conocimientos médico-físicos junto con determinada concepción de persona y cultura que asocian a la perforación.

Una de las principales formas de acceder a los procesos de autoidentificación social y personal es analizando los significados que las cosas culturales poseen para los actores, para así comprender las autovaloraciones y nociones constitutivas del nosotros (Barth 1976, 2005; Becker 2012).

Por ello, comenzar el abordaje reconstruyendo el período en que los actores tienen el primer contacto con estas prácticas corporales, para comenzar a interiorizarse en el oficio hasta convertirse en ejecutantes profesionales, resulta un modo deshistorizar los fenómenos sociales dentro de cada trayectoria individual. Ello nos permitirá reconstruir el tipo de ingreso transitado por cada sujeto y, como correlato, la consolidación de características particulares de los espacios comerciales de modificación corporal que en el caso de la ciudad de Santa Fe, caracterizado por su desarrollo reciente.

En este sentido es que destacamos, por un lado, las similitudes en las experiencias subjetivas de tatuadores y perforadores en lo tocante a su incursión por los oficios, como situaciones donde se transgreden las expectativas familiares y sociales vinculadas a sus cuerpos y especialmente a sus ocupaciones, moralmente sancionadas, vivenciadas como un distanciamiento social y familiar. Para luego destacar las particularidades de las prácticas de modificación corporal que realizan a otros, en donde se observan algunos de los significados centrales que atribuyen a estos oficios, como modos de vida.

Así, reconocemos dos situaciones. Un primer grupo formado por algunos de los tatuadores más antiguos de la ciudad, ingresaron en el mundo de las modificaciones corporales incursionando sin mediadores a una edad temprana (18 años aproximadamente) y aprendieron los trucos del oficio de forma autogestionada, a través de relatos de viajeros y del ensayo y error, por lo que se consideran autodidactas. Este subgrupo de tatuadores comparte la característica de que en vez de ingresar al sistema educativo entre los años 1992 y 1996 comienzan a trabajar en el oficio.

Los tatuadores más jóvenes, por el contrario, experimentan el tránsito y la conversión en el oficio luego de la deserción por las instituciones de educación superior, en simultáneo a un contacto temprano (adolescencia y juventud) con el ambiente de los tatuadores autodidactas asentados. Una vez que comienzan a frecuentar los locales de tatuajes y en posesión de la habilidad artística, resultan apadrinados por los más antiguos y comienza el proceso de enseñanza y aprendizaje de cuestiones técnicas y teórico-estéticas característico del vínculo entre maestro y aprendiz. 
El caso de los perforadores analizados pertenece a la generación de jóvenes, y según relatan presentan también la lógica de inserción en el oficio, tanto en términos de experiencia de ruptura, como concretamente, a por medio del contacto y tránsito por lo locales establecidos en donde comenzaron a interesarse por las perforaciones, para luego dedicarse de lleno. Inclusive, los primeros tatuajes que se realizaron (antes que las perforaciones), fueron realizados por uno de los tatuadores más antiguos, Gabriel, que forma parte de nuestro trabajo.

Podemos decir que existen dos generaciones de modificadores corporales, que se solapan con los dos tipos de ingreso al oficio mencionados. La primera generación compuesta de autodidactas son los que configuran el espacio formal de los tatuadores profesionales a nivel local, a través de la colocación de locales comerciales y generando ambientes urbanos de socialización donde acceder a estos servicios de forma pública y masiva.

Una vez establecidos, esta generación enseña el oficio a los jóvenes, que contarán con ámbitos de sociabilidad para interactuar con pares y consumidores y les proporcionan guías para el aprendizaje y la práctica. De este modo, en función de un recorte sincrónico evidenciamos elementos específicos, prácticas, representaciones y espacios, que permiten elaborar una diferenciación analítica al interior del grupo de modificadores corporales entre tatuadores y perforadores.

A través de un abordaje diacrónico, accedimos a la segunda característica de los oficiantes, la existencia común de dos generaciones de oficiantes basadas en el período y tipo de ingreso en este espacio de oficios, dando como resultado dos lógicas de introducción diferentes, como autodidactas o a través del vínculo maestro-aprendiz.

Al igual que Braz en torno a San Pablo y Costa para Florianópolis, el mundo de las modificaciones corporales en tanto problemática urbana, ocupacional y cultural evidencia la existencia de un corte generacional como principio estructurador (Braz 2005: 171; Costa 2004: 15), que se solapa con las dinámicas de iniciación y enseñanza mencionada.

La narrativa de los actores construye una historia compartida sobre el surgimiento de estas prácticas corporales y su ingreso en el mercado de consumo masivo, a través del proceso de autonomización relativa ${ }^{7}$ de los entornos informales (de su ejecución artesanal en los hogares) o de grupos sociales específicos (como los marineros o los presos), lo que delinea un modelo de la sociogénesis de estas prácticas como bienes de consumo cultural y de los sujetos como ejecutantes profesionales del oficio (Elias 1993, 1998) basado en el estudio del caso particular de la ciudad de Santa Fe.

\section{LA EXPERIMENTACIÓN COMO FORMA DE APRENDIZAJE}

Una cuestión que guía nuestro trabajo, surgida durante el contacto con los actores y que se sintetiza en la cita debajo, es que los modificadores conciben y construyen su actividad laboral y a las prácticas corporales que ejecutan como oficios, por lo que resulta una categoría nativa que retomamos como eje central del análisis.

Gabriel -Y bueno, empecé a laburar pensando que sabiendo dibujar era lo mismo y si bien es una...prácticamente un requisito, una necesidad, saber dibujar o tener facilidad para dibujar, porque estas dibujando, esto es un oficio que hay que aprenderlo, y después que lo aprendes es como que aplicas lo que vos dibujas viste...pero que no es lo mismo.

(Tatuador, 35 años). 
Esta clasificación compartida, implica un consenso y una homogeneidad acerca de sus autodefiniciones y constituye una condición de su existencia como grupo y de su diferenciación de otros modos de ejercicio de estas prácticas corporales. Así, como remarca Braz, tanto portar estas marcas como hacer de ellas una ocupación, producirá clasificaciones y jerarquizaciones hacia afuera en relación a su contexto social y familiar, como hacia el interior de los oficios (Braz 2005: 175).

Son percibidas como oficios, en primer lugar, porque su principal característica es que los conocimientos que implican en ambos casos son eminentemente prácticos, experimentales, esto es, no están atravesados por una educación escolarizada tradicional.

Por ende, remarcan que existen diferencias sustanciales entre un saber teórico (conocimientos médicos, teoría del arte, etc.) y el saber hacer. Este último no constituye un cúmulo de recetas, sino que involucra un manejo integral y una especialización de ese tipo de producto - el tatuaje o la perforación - y de su proceso de producción (Monteiro Leite 1996: 64).

Eso, sin embargo, no implica que estas prácticas modificatorias no posean técnicas específicas o lógicas de funcionamiento y aplicación, como tampoco que no estén sujetas a una especialización o perfeccionamiento, a través de la adquisición de conocimientos teóricos-técnicos sobre cómo realizarlo de la mejor manera, o por medio de los avances tecnológicos en esos campos o áreas vinculadas.

En segundo lugar, y en especialmente para el caso de la ciudad que analizamos, es un oficio porque su reproducción depende de formas de enseñanza y aprendizaje basadas en la transmisión oral, manual e informal (Busso 2006: 24), encarnada tanto en el vínculo maestro - aprendiz, o en la imagen del autodidacta, cuando los sujetos se interiorizan de manera autogestiva, pero que mantiene aún la modalidad informal y manual de aprendizaje.

Gabriel- Con el tatuaje también, fui bastante autodidacta, me paso que....cuando empecé a tatuar no había nadie acá para consultar para aprender, entonces eso era un problema...(...) a mí me pasó que como no había nadie no me quedaba otra que ir experimentando.

(Tatuador, 35 años)

La oralidad como forma de transmisión de saberes desempeña una función elemental en el oficio de tatuador, en torno a las técnicas utilizadas, la enseñanza de las estéticas históricas y escuelas artísticas, y asimismo, en lo relativo a la transmisión de los orígenes sociales y culturales de estas prácticas corporales, como el origen histórico y social marginal (alrededor de los marineros y los presos), o los conocimientos importados del ámbito de la cultura artística oficial, como teoría del arte.

Entre los perforadores el esquema resulta similar, pero se evidencia un interés y una movilización mayor de esfuerzos en la adquisición de conocimientos técnicos y teóricos a través de convenciones, workshops, talleres y cursos de especialización, como versa la cita. A diferencia de lo que reconoce Costa (2004) respecto de los tatuadores de Florianópolis, aquí el interés por las instancias organizadas de intercambio y aprendizaje son los perforadores los más interesados, mientras que los tatuadores parecen desestimarlas. 
Emiliano- Si yo doy un workshop, por ejemplo, diría que todos los que están haciendo el workshop se tienen que perforar, tienen que saber cortar, van a vivir en carne viva el procedimiento, porque lo tienen que ver, yo no te puedo corregir algo si no lo veo como lo haces, entonces es simple yo no te lo puedo decir porque no vas a entender nada...

(Perforador, 26 años)

Vemos en la enseñanza manual un importante mecanismo de aprendizaje de estas prácticas y del perfeccionamiento de la técnica como pilares de la profesionalización, y como dice el entrevistado, ejecutarla sobre uno mismo conforma un requisito de comprensión de la práctica misma.

En cuarto lugar, la lógica de consagración y legitimación dentro del ámbito no deriva de un sistema de titulaciones sino de habilidades manuales, sean artísticas o provengan de la posesión de conocimientos fisiológicos sobre el cuerpo. Por ambas razones, la trayectoria de formación entendida como experiencia y la antigüedad en el oficio serán elementos determinantes de la legitimidad en este espacio social.

Asimismo, los entrevistados entienden a sus prácticas modificatorias como un oficio porque es posible construir los utensilios o maquinarias necesarias de forma artesanal, otorgándoles a los iniciados margen de libertad ante los intermediarios económicos, que luego serán centrales para el comercio oficial de la práctica, como la figura del proveedor. Actualmente, hay un sinnúmero de medio informativos que enseñan a construir máquinas artesanales para iniciarse en la actividad, por la inversión inicial que supone comenzar a tatuar con máquinas profesionales.

La naturaleza del producto derivado de competencias artísticas o artesanales, es decir como productos originales e irrepetibles, permite comprender también estas prácticas como oficios, en tanto constituye una de las clasificaciones centrales para los sujetos.

En último lugar, el argumento más significativo es que estos oficios son vivenciados por los actores como algo que va más allá de la supervivencia material para abarcar una forma o filosofía de vida (Busso 2006: 24), que se materializa en prácticas corporales pero que implica una cosmovisión del mundo, significados compartidos, herencias y tradiciones.

De este modo, la articulación de experiencias, adhesiones corporales y discursos de transgresión, estructurados desde su adscripción al oficio, y en función de decisiones vitales autorelevantes, permiten comprenderlos como estilos de vida (Giddens 1991: 106), en tanto definen de este modo sus principales coordenadas identitarias.

\section{CONVERSIÓN AL OFICIO: DISTANCIAMIENTO SUBJETIVO Y PROFESIONALIZACION}

Como dijimos, el tatuaje es una marca permanente en la piel realizada con pigmentos que, a diferencia de las perforaciones, se materializa en dibujos o diseños en color o en blanco y negro, lo que le otorga funciones expresivas y artísticas, privativas de esta práctica corporal.

Según los relatos, pudimos construir tres modalidades de ejercicio del tatuaje en función de las características específicas del entorno, los recursos técnicos y habilidades artísticas, el contexto social y la simbología de los diseños: el tatuaje artístico, que se subdivide en 1) el tatuaje artístico-artesanal 2) el tatuaje artístico-profesional; y por último el 3) el tatuaje carcelario ${ }^{8}$. 
Los tipos mencionados son tatuajes característicos de la sociedad moderna y encajan dentro de una concepción del tatuaje como adorno del cuerpo, en tanto este último es factor de individuación, posee la función de medio diferenciador por que "ocupa puntos diferentes del tiempo y del espacio" (Durkheim 1968: 277). Actualmente, el cuerpo estará abierto a las decisiones reflexivas y autorreferenciales de los individuos que, en el marco de los procesos de individualización contemporáneos, colocan al sujeto como agente responsable del proyecto identitario (Giddens 1991:128).

Las conceptualizaciones o tipologías que derivan de la descripción mencionada denotan la posesión de sistemas clasificatorios comunes constitutivos del oficio de tatuador, formas de comprender su práctica que forma parte de la acción misma y de su forma de vida, hecha cuerpo (Bourdieu 1986, 2012).

El ingreso y aprendizaje al oficio de tatuador se produce a través de lo que reconocimos como tres etapas estereotípicas que atraviesan los ejecutantes, clasificadas como contacto, iniciación y profesionalización, con sus variantes para cada trayectoria individual, expresando lo que podemos entender como un proceso de conversión, en tanto sintetiza transformaciones subjetivas y biográficas fundamentales.

Esta división analítica no denota etapas perfectamente demarcadas en la biografía de los sujetos, sino que busca construir límites ideales derivados de una sucesión de acciones, decisiones y de la circulación por espacios, que se entrelazan con la vida personal de los entrevistados. Las fases mencionadas aluden a los momentos que transitan hasta establecerse como profesionales, que tienen como punto culmen la comercialización del servicio.

La fase de contacto constituye, en términos generales, el acercamiento inicial con este ámbito de prácticas, momento en el que conocen a los tatuadores y comienzan a transitar de manera asidua los locales, interactúan con los oficiantes, observan lo que hacen, aprecian los trabajos, etc.

Este momento se encuentra marcado en la biografía por la primera modificación corporal que deciden realizarse los sujetos, que adquiere tres significados importantes en el marco de su trayectoria personal, convirtiéndola en un hito dentro de la misma.

El primer lugar, consuma el primer contacto con este ámbito, constituye la decisión de acercarse, elección que algunos de los entrevistados reconocen como el inicio de la pasión y/o vocación por el oficio. En segundo lugar, este hito adquiere importancia porque es la etapa de conocimiento del maestro, central para la reproducción del oficio, que generalmente es quién les realiza esta primera modificación.

Por último, autorealizarse el primer tatuaje resume, según mencionan, un cambio subjetivo e identitario importante porque sintetiza el momento de distanciamiento o ruptura con el entorno familiar (por el rechazo que generan estas prácticas) y las consecuencias o experiencias sociales que supone portar una marca corporal de esta envergadura en el marco de la sociedad santafesina, culturalmente cerrada para los entrevistados.

En la segunda etapa, de iniciación, la persona con la que tienen contacto inicial comienza a enseñarles oralmente cuestiones relativas al tatuaje, a las técnicas y a las tendencias artísticas, y simultáneamente se van embebiendo del escenario y aprendiendo mientras observan cómo otro tatuador realiza estas prácticas sobre los clientes. 
Fabricio - A los 16 años hice mi primer tatuaje, era un desastre comparado con ahora. Le agradezco mucho a los amigos que confiaron en mí, para que los tatúe...Todavía siguen viniendo a que les arregle lo que les hice, gratis para no quedar mal viste, sino van a andar con eso les preguntan y dicen "Fabricio", no da...

(Tatuador, 32 años)

Como dice la cita, en este momento comienzan a realizar tatuajes de manera artesanal, gratuita y esporádica sobre amigos y familiares en sus hogares, por lo que reconocen y agradecen a las personas que se ofrecieron al inicio de sus trayectorias a que prueben, ya que de otra manera hubiese sido dificultoso aprender sin experimentar reiteradas veces en piel humana9.

En las narrativas de conversión este periodo resulta central porque es el eje articulador entre el tatuador profesional y el tatuador de barrio, como establece el entrevistado. Esta categoría nativa es utilizada para calificar, en primer lugar, a los sujetos que tatúan de forma artesanal en sus hogares, lo que denota falta de experiencia en la práctica de tatuajes artísticos. Esta inexperiencia y la irreversibilidad que caracteriza a los tatuajes como marca corporal, sobrelleva la imposibilidad de cobrar por los mismos.

Juan- Empecé a hacerlo, y ese tatuaje me quedó bárbaro, o sea era el primer tatuaje que yo decía “bueno este es un tatuaje", el primer tatuaje que yo decía esto es un tatuaje profesional, esto es lo que puedo mandarme y hacérselo a cualquier persona, entendés, y no va a haber ningún problema y me lo va a pagar como si fuera a cualquier local a hacérselo, o sea me podía lanzar comercialmente, si llegaba a hacer bien eso.

(Tatuador, 24 años)

En segundo lugar, alude a personas que no se han establecido comercialmente $y$, si lo han hecho es de forma artesanal, lo que en ambos casos alude a los primeros estadios de la experimentación, y claramente a la falta de profesionalización. El eje aquí es que en general no salen del margen del barrio hacia zonas más comerciales de la ciudad para situarse a la altura de los tatuadores profesionales, proceso caracterizado como de traslado de la "periferia al centro" (Costa 2004: 24).

Juan - Sin desmerecer a los tatuadores de barrio porque todos fuimos tatuadores de barrio, ¿no? Pero le podes hacer lo que quieras y no va haber ningún drama, pero ahí está en cómo querés hacer vos tu nombre (...).

(Tatuador, 24 años)

La cita mencionada sintetiza un sistema de clasificación, o como denomina Braz, la normativización del campo que produce jerarquizaciones hacia su interior, caracterizado por la posesión de un aparato de inteligibilidad (2005: 174-175). En nuestro caso, se compone de dos elementos (habilidad-experiencia y comercialización) fundamentales para el establecimiento, el prestigio y la legitimidad de los tatuadores profesionales, que significa esencialmente renombre en torno a las capacidades para tatuar, lo que deriva en la posibilidad de cobrar.

Según los relatos, todos atraviesan esta etapa en los primeros pasos de construcción de la trayectoria, que se caracteriza por un período de acondicionamiento, a la necesidad y posibilidad de experimentar sobre las pieles para adiestrar la mano, porque ello condiciona la calidad del tatuaje artístico-profesional, de índole estética. 
Generalmente está acompañado de la adquisición de los elementos necesarios (herramientas, maquinarias y materiales) para realizar tatuajes de buena calidad y puede o no estar acompañado de la colocación de un local, lo que dependerá de la autopercepción del manejo de la técnica por parte del oficiante y de sus recursos a disposición.

Una vez que probaron de manera artesanal y que se sienten seguros con la mano corroboran, por medio de la realización de un tatuaje que consideran un desafío artístico, que alcanzaron un nivel de manejo de la práctica que los habilita a realizar cualquier diseño que se presente y que se hallan preparados para cobrar por sus prácticas. Están en condiciones de introducirse en el comercio oficial y profesional de tatuajes.

En este sentido, vemos que el comercio oficial y la profesionalización van de la mano, se suponen, en tanto la práctica hace a la mano, al adiestramiento que lleva a la profesionalización, indisociable de la habilidad y las herramientas que licencian la comercialización.

Por último, la etapa de profesionalización conlleva el comercio del servicio, por lo que el tatuador vende sus aptitudes y realiza tatuajes artísticos, como profesional. En términos de expectativas profesionales, el establecimiento de un local propio es la principal proyección en el ámbito analizado. Estas aspiraciones son expresivas tanto de las trayectorias de los tatuadores como de los perforadores, en lo tocante a las expectativas.

Algunos tatuadores comienzan trabajando para un tatuador con un local ya establecido, de relativo renombre, que va compartiendo y/o relegando trabajos en el neófito como un mecanismo de adquisición de experiencia y también para colocar el estilo estético del tatuador empleado en el mercado de tatuajes.

\section{ARTE Y COMERCIALIZACION: LOS TATUADORES}

Un modo de comprender el alcance del ejercicio del oficio para los tatuadores, es reparar en la centralidad que otorgan al haber pintado y/o dibujado desde pequeños. Reconocen que es la actividad que hacen mejor y que más les gusta, otorgándole un carácter autodefinitorio porque según dicen nacieron dibujando.

Esto permite ver, en su relacionamiento, un sinnúmero de decisiones y elecciones -en apariencia independientes- como ser las instituciones de enseñanza media a las que asistieron (por ejemplo, de terminalidad artística) o los consumos culturales a los que se han abocado.

En algunas de las trayectorias analizadas, explican esta aptitud y este gusto acentuando su relación con las ocupaciones y habilidades heredadas de los padres, aludiendo al tránsito de su niñez y adolescencia en un entorno artístico, como ámbito simbólico de formación emocional, intelectual y personal.

La lógica de aprendizaje y apropiación del oficio se desarrolló con posterioridad a su vocación artística, ya que todos son pintores amateurs que luego se abocaron a incursionar con el oficio de tatuador.

En este sentido es que los actores se autoperciben y definen como pintores o dibujantes, entendiendo la primera expresión como pintura al óleo y la segunda como la actividad de dibujar con lápiz o crayones, por ejemplo Comics. Aunque estas actividades sean intercambiables, en general se abocan a una de ellas por cuestiones de habilidad manual y artística, de afinidad con los estilos y las tendencias estéticas, siendo un criterio de definición y clasificación en el oficio. 
Las predilecciones y aptitudes del tatuador los van perfilando hacia uno u otro estilo, en el cual se perfecciona a lo largo de la trayectoria y, cuando los diseños personales se colocan en el mercado y el estilo artístico del tatuador se convierte en su marca personal, comienza el proceso circular de renombre, ejecución, reconocimiento.

Por lo mencionado, los sujetos entrevistados se sienten artistas, pero construyen una distinción fundamental entre el artista tradicional y el ejercicio del arte en el contexto del oficio de tatuador, basado en el hecho de que su lienzo es la piel. Esta transposición simbólica representa la ruptura hacia sus contextos subjetivos y objetivos, el entorno familiar y hacia las instituciones oficiales.

Los tatuadores coinciden en que saber dibujar (habilidad artística) es un requisito, una necesidad para ejercer la actividad, pero que no necesariamente alcanza. El núcleo del oficio radica en el proceso de adaptación a dibujar sobre la piel, basado en la práctica y la experimentación progresiva de tatuar, saber realizar diseños sobre piel y no ya en papel. Así, cuando aluden a la profesionalización remiten a la necesidad de manejar ambas facetas: la capacidad artística y la experiencia de tatuar sobre piel, como establece el entrevistado.

Las prácticas de los sujetos se comprenden sobre el telón de fondo del proceso de individualización, dada la autoreferencialidad interna en el reconocimiento de ideas, valores y emociones subjetivas (Giddens1991: 104), de las que los sujetos dan cuenta reflexivamente cuando discutimos acerca de las prácticas corporales que se autorealizan.

A estas afirmaciones no sólo subyace una cuestión de ruptura. Se comprende que los sujetos articulan las habilidades que poseían dentro de una estrategia material, ya que no se hallaban exentos de la urgencia económica.

Los tatuadores analizados coinciden en que el inicio de la actividad fue no sólo por una cuestión contestataria, sino que la visión rupturista se conjuga con razones económicas, en tanto explican su ingreso al oficio de tatuador porque resulta una forma rentable de ejercer el arte, una actividad productiva que les garantiza la subsistencia haciendo lo que más les gusta.

Ahora bien, una vez que finaliza la etapa que caracterizamos de profesionalización, que representa la venta del servicio de forma oficial, los entrevistados comienzan a sentir las presiones y limitaciones vinculadas al comercio de la actividad.

Para los tatuadores, el servicio que ofrecen genera, algunas veces, la sensación de falta de libertad porque deben someterse a las necesidades de los clientes, muchas veces supeditados a los movimientos de la moda. A diferencia de la noción de "trabajo perfecto" que Costa (2004: 69) resalta en su trabajo sobre tatuadores, en nuestros relatos los sujetos asocian su práctica artística con ideales vinculados a libertad de creación y producción original.

Así, además del estigma que caracteriza al oficio de tatuador en el marco de la ciudad, también las presiones y los tipos de demandas vinculadas a la comercialización de sus producciones, producen tensión entre las autodefiniciones de los actores, basadas en la noción de artista libre característica de la figura de dibujante o pintor (Becker 2012: 129). 
A pesar de las restricciones mencionadas, existen momentos en los que sortean esos límites en lo que uno de los entrevistados denominó freehand (manos libres), que supone realizar un dibujo libre de ataduras, sin boceto ni diseños previos, aunque puede haber líneas o estéticas tentativas, el dibujo queda al albedrío del tatuador. En el polo contrario, están los que se acercan sin saber en absoluto qué es lo que desean, situación que tampoco los conforma ya que en algún sentido no saben por dónde comenzar.

El contacto temprano con el mundo de las modificaciones corporales -especialmente a través del los tatuajes -, conjugado con habilidades artísticas y la negativa de insertarse en una institución educativa, en un contexto marcado por la necesidad de comenzar a realizar una actividad económica, explican el ingreso y comienzo en el oficio.

Vemos entonces que los tatuadores comparten sistemas clasificatorios constitutivos a la práctica del oficio, que se comprenden en función de características comunes de las trayectorias, de una historia social compartida y de la pertenencia al espacio social a nivel local. A través de la reconstrucción de lasociogénesis de esta práctica en el marco local, esbozamos las diversas aristas que conforman este espacio: la comercial y la artística.

En base a eso pudimos ver que los pilares de la trayectoria son, por un lado la antigüedad, vinculada a los primeros locales comerciales y a los primeros tatuadores establecidos en un espacio físico y social limitado, que se comprende también en función de la relación entre las generaciones; el segundo pilar, es la mano individual de cada tatuador (la habilidad artística), su experiencia y su estilo, lo que viene a explicar su colocación y perpetuación en el mercado.

\section{EL OFICIO DE PERFORADOR COMO FILOSOFÍA: EL BUEN PERFORADOR}

Durante la salida al campo, observamos que en los locales comerciales abordados en general se realizaban tanto tatuajes como perforaciones, ejecutadas por personas distintas, aunque dentro del universo encontremos excepciones donde se ofrece sólo una de las prácticas modificatorias.

Los perforadores o bodypiercers entrevistados presentan diferencias con los tatuadores, como mencionamos, pero no justificaron la construcción de un modelo de conversión al oficio independiente al de los tatuadores.

Aquellos experimentaron de forma análoga el proceso de ruptura y distanciamiento temprano con el entorno familiar y social reconstruido en torno a los tatuadores, donde la ruptura inicial comienza con la realización de tatuajes en la adolescencia, para profundizarse luego cuando se ejecutan otras modificaciones corporales, como piercing, expansores, suspensiones, y finalmente cuando se inician en el oficio de perforadores.

La evolución de la práctica de la perforación parece haberse desarrollado de manera anexa y dependiente del mundo de los tatuajes, lo que podría explicar el esfuerzo explícito de los entrevistados en la construcción y el mantenimiento de límites grupales exclusivos, operacionalizados través de determinada concepción de perforador, por lo menos en el ámbito local.

Las narrativas se centran en la construcción de una definición de un tipo ideal de perforador identificado con ellos mismos, dejando en claro que pueden observarse en el espacio analizado algunos perforadores que no se ajusten a la distinción construida por los entrevistados. Esto se funda en la caracterización contrastante entre la forma de concebir el trabajo de los competidores locales y la forma propia, mejor y diferente. 
Esta diferencia con los otros perforadores locales radica no sólo en que no realizan la totalidad de las perforaciones y modificaciones posibles que podría realizar un perforador (branding, escarificaciones, etc.) sino, especialmente, en que no las realizan de la "forma correcta", es decir no entran dentro de la categoría de buen perforador.

Para los perforadores entrevistados el oficio que encarnan es parte de una definición amplia que entiende a la perforación como forma de vida. En la cita, el entrevistado busca realizar una fuerte distinción entre los que poseen una concepción englobadora de la perforación, como filosofía o forma de vida, donde las prácticas modificatorias y otros consumos (como el ejemplo de piezas antiguas) adquieren un significado diferente a la de un mero producto comercial o estético.

Emiliano - Nosotros siempre hablamos - aclara - que por ahí es medio discriminatorio, un verdadero modificador o verdadero bodypiercer que vive el piercing como cultura no es un tipo que vive con los aros de ahora o sea yo tengo piezas, que en realidad no las uso porque son antiguas y no tengo las plata para comprarme cosas, pero la mayoría somos fanáticos de las tribus y armamos joyas tradicionales, nada de aros nuevos con loguitos, buscamos cosas bien tradicionales madera hueso bronce cosas bien...ninguno, más allá de que podamos usar zapatillas, mp3, lo que vos quieras, porque me ha pasado encontrarme, yéndome a Francia me encontré con un amigo que también es modificador y los dos estábamos y como que, mp3 y todo y el tenia unas joyas de madera que tenían 100 años cada una que las trajo de Perú que se las había comprando a un indio en Perú y le había regalado unas gemas que estaban buenísimas, y somos medio por ese lado como que y siempre hablamos el verdadero el perforador de alma, no te digo que este mal ser comerciante, pero el nuevo...es esa la diferencia, no va a tener una banda de punk rock y un par de aros, lo disfrutas por otro lado como forma de vida... todo flahseamos de que como no vivimos hace 100 años en Tailandia, con el auge de todo eso es como que decimos la puta madre vivimos ahora cosas viejas tenemos más espíritu retro, en ese sentido, somos más indios.

(Perforador, 26 años)

Como la tarea del perforador es realizar prácticas corporales, consideran que las personas constituyen no sólo un cuerpo sino una entidad orgánica y compleja que posee una psicología que aflora a través de diversos mecanismos, enarbolando una concepción relativamente holista del individuo.

A diferencia de los tatuadores, el eje de la construcción identitaria de este grupo de perforadores se fundamenta más en una cuestión de ideas, valores, formas de vivir y experimentar la práctica, que en la adhesión a una historia compartida y en la consolidación espacial, temporal y artística del oficio.

La concepción del buen perforador tiene como primer elemento definitorio la disminución y control del riesgo, que se ajusta y/o deriva de su forma de ejercer el oficio. Ser buen perforador significa realizar sus prácticas con los recaudos (cuidados e higiene) necesarios asegurando primeramente la disminución de los riesgos de las prácticas invasivas ejercidas sobre los cuerpos, lo garantiza un buen trabajo.

Esta concepción del buen hacer se relaciona con las dificultades particulares de esta práctica modificatoria. Los sujetos mencionan que existen riesgos relativos a enfermedades de transmisión sanguínea, como puede ser HIV o Hepatitis, y que descuidos vinculados a la esterilización pueden desembocar en el contagio por parte de los clientes ${ }^{10}$. 
Argumentan que existe la probabilidad, en algunas de las prácticas, de equivocarse o caer en el error dada la complejidad y el comprometimiento del cuerpo, aun siendo un perforador con experiencia y trayectoria internacional reconocida, por eso los resguardos deben constituir un eje directriz del ejercicio del oficio.

Algunos de los recaudos son, por ejemplo, reparar en el estado general de los consumidores cuando se acercan a realizarse modificaciones como una política de trabajo del perforador, examinar el grado de conciencia que tienen sobre lo que están demandando y el estado anímico en el que se encuentran, porque es una práctica que impresiona e impacta y a la cual los consumidores a veces reaccionan negativamente, desmayándose, retractándose o conmocionándose a un punto que impida ejecutarla.

Por otro lado, ser buen perforador implica también la capacidad de elaborar un proyecto estético, que significa la habilidad de construir una determinada estética, que exprese simetría, equilibrio, prolijidad, sobriedad y que se entiende como la forma correcta de hacer las cosas, como el objetivo primordial del ejercicio del oficio ${ }^{11}$.

Esta concepción del hacerse contrapone, en el discurso, tanto al "hacer por hacer" de otros perforadores que sólo buscan vender, como también al tipo de consumidor que busca inevitablemente realizarse modificaciones sin una idea estética previa, de lo que buscan o de lo que obtendrán estéticamente al realizárselas.

El desafío no reside en la colocación mecánica, sino en la capacidad de realizar un proyecto estético acorde al consumidor, en dos sentidos: en primer lugar, siempre debe curar apropiadamente; por otro lado, debe quedar bien sin atiborrar a la persona y realizarse en función del estilo general, es decir, proyectar algo que esté en línea con la estética del consumidor, como parte de una armonía del sujeto en su conjunto.

Resulta sinonímico el vínculo entre esta noción de construcción estética basada en la idea de estilos como unidades con coherencia relativa entre sus partes y los significados que otorgan a la perforación como forma de vida.

Por último, el relato deja ver que la concepción del buen perforador significa sujeción y respeto a las normas legales de ejercicio del oficio. Aquí se analizan los vínculos entre las nuevas disposiciones legales que regulan la actividad de la perforación y su comercialización. Como ocurre en otras actividades a veces el que hace las cosas bien, entendiendo por ello las cosas en regla (en función de regulaciones), resulta perjudicado comercialmente, según la percepción de los actores ${ }^{12}$.

Eso ocurre según los entrevistados porque, en términos de costos, es menos rentable ajustarse a las reglas porque algunos clientes que demandan estas prácticas buscan precio en el servicio, es decir, prefieren ir en detrimento de la calidad por menos costo.

\section{TIPO DE PERFORACIONES}

Como ya mencionamos, el oficio analizado se basa en la realización de perforaciones en diferentes partes del cuerpo, de formatos variados, estilos estéticos disímiles, que varían en torno al grado de comprometimiento del cuerpo, como también de la complejidad de la colocación y de la dificultad de la cicatrización. A ello se suma, el tipo de experiencia corporal que se busque con la modificación y el resultado obtenido. 
Manuela: si, muchos son para estética y otros son para placer. Unos que le dan placer a la mujer y otros que le da placer a la otra persona...

(Perforadora, 25 años)

Dentro de las modificaciones que realizan los oficiantes, existen prácticas que no son extremas (en el sentido de riesgosas) pero que no resultan convencionales. Indagando en diversas fuentes observamos en la red prácticas modificatorias realizadas por algunos de los entrevistados que forman nuestra muestra, en zonas inusuales del cuerpo como los genitales femeninos y masculinos.

Como deja ver el fragmento de entrevista, cuando consultamos sobre el objetivo de tales modificaciones para saber cuáles eran los móviles de las mismas, accedimos a otro sentido subjetivo acerca de las perforaciones: el placer. Más aún, los piercing en las zonas genitales no sólo tienen como finalidad de la acción el placer propio, del que se las práctica así mismo, sino también el placer del otro durante la relación sexual.

Podemos ver que los nombres de los piercing y su historia (por ejemplo el piercing denominado Príncipe Alberto) son parte del conocimiento común de los oficiantes, al igual que el conocimiento del trasfondo históricosocial de los tatuajes. Ello expresa, más allá de las diferencias identitarias, la posesión de sistemas clasificatorios compartidos por los perforadores y, en términos amplios, por los consumidores regulares de estos bienes específicos.

Durante la observación en el local, a través de books de los oficiantes, de los productos que se ofertan en las vitrinas, y en función de los diálogos entre los clientes y los perforadores, pudimos establecer que las zonas del cuerpo donde se ejecutan las perforaciones son: Cejas, Nariz, Tabique Nasal, Oreja, Mejilla, Labio, Ombligo, Lengua, Genitales Masculinos y Femeninos, Pezones, y algunos en partes llanas del cuerpo, como Pecho, Muñeca.

Al igual que en el ámbito de los tatuajes, los demandantes de estas prácticas a veces recurren a realizarse cosas en el cuerpo que no son técnicamente realizables o no son recomendadas por los perforadores, porque no cicatrizará bien o porque compromete demasiado la zona del cuerpo, lo que deja librado al consumidor un proceso de curación complejo, comprometiendo demasiado el producto y, por ende, el renombre de los ejecutantes.

Hacia adentro del oficio la cuestión se complejiza dando lugar a un abanico de prácticas de modificación corporal que se aleja y extrema de los piercing tradicionales. Generalmente estas modificaciones son realizadas por perforadores comunes que luego se profesionalizaron en estas prácticas antiguas actualmente comerciales, como los branding y escarificaciones.

Igual, aunque sepan ejecutarlo -como se ve con los entrevistados- no necesariamente desean practicarlo a otros o a sí mismos, en tanto el grado de aumento de la transgresión del cuerpo que suponen estas prácticas es elevado, ampliando el riesgo físico de forma sustancial.

Al aumentar el comprometimiento del cuerpo, los entrevistados concuerdan en que es preferible realizar cosas que estén dentro de los objetivos previstos, antes que someterse a realizar desmedidamente prácticas que pueden ser fisiológicamente riesgosas pero que se realizan porque se ponen de moda en determinados grupos o nichos de grupos. 
Como vemos, la extremación de las prácticas de modificación corporal poco convencionales, podría responder al mismo proceso social de construcción de la diferencia que simbolizaron socialmente los tatuajes y las perforaciones, de forma inicial cuando buscaban expresarse como símbolos subversivos o contraculturales, para determinados grupos sociales. Aunque algunos autores remarcan que este interés diferenciador responde a lógicas individualizadas antes que grupales, aunque la producción identificatoria sea igualmente un objetivo.

Se podría entonces inducir que ello engendra la pérdida de la función fronteriza que antaño podía desempeñar en algunos nichos sociales, debiendo generar dentro del campo de las modificaciones corporales, otras prácticas que denoten la exclusividad que los entrevistados buscaban en un inicio con las perforaciones tradicionales, por lo menos en el contexto de la ciudad de Santa Fe.

Por ello su surgimiento debe leerse sobre el telón de fondo de dos procesos vinculados pero que podrían indagarse de forma independiente, por un lado, la asimilación de estas prácticas a la cultura de consumo como consecuencia de su progresiva masificación, como característica de la novedad (Simmel 2002: 55-56). En segundo lugar, como expresión de los procesos de individualización contemporáneo, donde la construcción estética y la autoreferencialidad serán componente centrales de los procesos de construcción de las biografías del yo y de los relatos de sí mismos (Lash y Urry 1997; Beck et al. 1997).

\section{CONSIDERACIONES FINALES}

Durante el desarrollo del análisis del oficio de tatuadores y perforadores encontramos que existen diferencias que, aunque permiten hacer distinciones hacia el interior, no justificaron un tratamiento exclusivo de estos subgrupos por separado.

Al recuperar la categoría de oficio, observamos que perviven las modalidades tradicionales de reproducción pero en oficios rupturistas o poco convencionales, por lo que resultaría interesante hacer dialogar algunos de los hallazgos derivados del ejercicio propuesto con la naturaleza de los procesos de aprendizaje en otros oficios.

Luego de reconstruir las razones nativas de porqué son considerados como oficios, observamos en sus ejecutantes la posesión de sistemas de clasificación compartidos, basados en un conjunto de ideas, valores y prácticas, cuyo eje será la experimentación como mecanismo de aprendizaje, modo adquisición de conocimientos prácticos basado en la transmisión oral y como componente central de la antigüedad y el prestigio dentro del oficio.

Se comprende también la trascendencia que adquiere en la vida de los sujetos la primera modificación corporal, los tatuajes, configurándose como un hito subjetivo.

En el caso de los tatuadores, existen formas de ejercicio del tatuaje que se corresponden con determinados tipos de tatuaje, erigidos en función de un criterio complejo que articula espacios, significados, estéticas y herramientas para la producción. Adeudamos con ello un análisis más profundo de las expresiones corporales y estéticas en contextos situacionales diversos (como el de encierro), en tanto el carácter contextual de estas prácticas adquiere sustancial relevancia. 
A través del análisis de las biografías, delimitamos las diversas etapas de conversión en el oficio hasta formarse en tatuadores artístico-profesionales. Estas etapas permitieron describir tres elementos que se presentaron como fundamentales para explicar las decisiones estratégicas de los sujetos en contexto y su oficio: los habitus artísticos, las autodefiniciones y el ejercicio del arte a través de espacios sociales alternativos a la cultura artística oficial.

En función de ello se comprenden las tensiones que se generan en torno a la comercialización del oficio en sujetos que se autodefinen como artistas y buscan vivir esa autodefinición en libertad y porque portan una cosmovisión del oficio como una forma de vida.

En el caso de los perforadores, estos elementos están condensados en los caracteres de lo que distinguimos con fines analíticos como el tipo ideal de perforador.

A través de la delimitación de este tipo ideal se describe a un tipo perforador que es esencialmente un buen perforador cuyos componentes articulaban cuestiones vinculadas a lo económico, lo fisiológico y sanitario, y la relación de esta práctica con los diferentes niveles del Estado a través de regulaciones al ejercicio del oficio.

Estos elementos distintivos son: el control/disminución del riesgo fisiológico de los consumidores, la formulación de un proyecto estético coherente con el perfil estilístico del consumidor, el respeto a las normas legales como buen hacer, y esencialmente, la posesión de una cosmovisión cultural de la perforación como forma de vida, basada en la persona como sujeto emocional y físico, que experimenta por el cuerpo. El entramado de relaciones, espacios y prácticas que se documentan con el oficio de tatuador resulta entonces sumamente prometedor para estudios ulteriores.

Vimos que el significado de estas modificaciones posee una importancia cardinal porque conjuga el cuerpo con formas de descontento que se sintetizan, en diálogo tenso con el contexto subjetivo y objetivo, en la elección de dedicarse a estos oficios, configurando de ese modo, biografías y trayectorias transgresoras.

Finalmente, podríamos pensar que las etapas y procesos implicados en la profesionalización de los oficios descritos, constituyen, por un lado, una descripción concreta del desarrollo del oficio en una configuración específica, la ciudad de Santa Fe. Pero también, estas etapas podrían conformar -en el marco del diálogo con los aportes de otros autores sobre procesos análogos- hipótesis de trabajo para el análisis de procesos de consolidación y profesionalización de estos oficios en diferentes entornos sociales, aun no abordados.

Melania Stehli es Doctoranda en Humanidades y Ciencias (Antropología Social) por la Universidad Nacional de Rosario (UNR), Becaria Doctoral del Consejo Nacional de Investigaciones Científicas y Técnicas (CONICET), y Jefa de Trabajos Prácticos con dedicación simple por la Universidad Nacional del Litoral (UNL). 


\section{NOTAS}

1 Ello se documenta en el desarrollo y ubicación de los locales comerciales o estudios en las grandes ciudades, como documentan los trabajos etnográficos realizados en ciudades brasileñas, citados en la bibliografía, a diferencia de su despliegue a nivel local, donde los locales se encuentran dispersos.

2 Aludimos con profesionales a los individuos que comercializan oficialmente el tatuaje y la perforación, teniendo luego criterios de profesionalización diversos en función de estándares internos de cada subgrupo.

3 Escogimos la zona comercial principal de la ciudad porque es donde se concentra la mayor cantidad de locales o "estudios". Para una descripción de estos espacios vea Zelia Costa (2004), quien realiza una etnografía pormenorizada de estos locales como espacios físicos y sociales, del caso específico de Florianópolis.

4 El fenómeno de la conversión busca sintetizar la transformación de componentes subjetivos (creencias, valores, ideas, emociones) por parte del actor, al igual que se utiliza en la religión, pero estableciendo una relación con los elementos objetivos al mismo, como remarcan Berger y Luckmann (2006) y Berger (1969). En nuestros actores, nos pareció que el término conversión resultaba expresivo del distanciamiento social y subjetivo que experimentan los oficiantes en el transcurso de su adhesión a estas marcas corporales, que se corona con el proceso de aprendizaje en un oficio.

5 Se realizó observación participante en los locales comerciales de los tatuadores y perforadores, como recurso central del reconocimiento de los mecanismos, lógicas sociales y dinámicas relacionales que no se aprehenden a través del discurso. El número de entrevistados es de 7 personas, 5 son tatuadores y 2 son perforadores profesionales de la zona comercial principal, cuyo acceso estuvo condicionado a la predisposición de los actores, resulta significativo que encontremos dentro de estas entrevistas algunos de los oficiantes más antiguos, y algunos de sus aprendices. De los tatuadores entrevistados, dos de ellos poseen 35 años, otro 32, mientras que los restantes poseen 28 y 24. Los perforadores tienen por un lado 26, y la única mujer tiene 25.

6 Las escarificaciones son escaras (cicatrices) producidas en forma intencional por cortes o quemaduras. El branding consiste en el hecho de marcar la piel por medio de hierro caliente, o a veces bisturíes calientes. Las expansiones es una modificación en la que se hacen aberturas o huecos en ciertas partes del cuerpo, como puede ser en la parte superior o inferior de la oreja (la más común), entre los orificios de la nariz o ya sea a su costado o en el labio inferior, estas son las más usadas.

7 Con proceso de autonomización aludimos a un proceso de construcción de un espacio de modificadores corporales comerciales, como actividad profesional con estándares internos propios desligado de las connotaciones de su origen social marginal. Esta actividad era un signo característico de un grupo social, realizado artesanalmente y en los hogares.

8 El tatuaje carcelario tiene vínculos con el tatuaje artístico casero, y hay estudios sociológicos y antropológicos vinculados al significado y a la función que desempeña en los entramados característicos de las situaciones de encierro y en los sectores populares.

9 Los entrevistados comentaron que anteriormente se practicaba con cuero de chancho o piel sintética, pero remarcaron las diferencias con la piel humana y el costo de comprar el material para practicar.

10 Este tipo de inconveniente sanitario es casi nulo en la actualidad, dado el avance en la oferta de materiales descartables y tecnologías de esterilización.

11 Podríamos decir que este énfasis en una coherencia estilística es una práctica muy común en los grandes consumidores de perforaciones.

12 Ver la Ordenanza Municipal de la Ciudad de Santa Fe n 11299. 


\section{REFERÊNCIAS BIBLIOGRÁFICAS}

BARTH, Frederic. 1976. "Introducción”. En: F. Barth (comp.) Los grupos étnicos y sus fronteras. La organización social de las diferencias culturales. México: Fondo de Cultura Económica.

2005. "Etnicidade e o conceito de cultura". Antropolítica: Revista Contemporânea de Antropologia e Ciência Política. 19 (2):15-30.

BECK, Ulrich \& BECK-GERNSHEIM, Elizabeth. 2003. La individualización. El individualismo institucionalizado y sus consecuencias sociales y políticas. Buenos Aires: Paidós.

BECK, Ulrich; GIDDENS, Anthony \& LASH, Scott. 1997. Modernización reflexiva. Política tradición y estética en el orden social moderno. Madrid: Alianza.

BECKER, Howard. 2012. Outsiders. Hacia una sociología de la desviación. Buenos Aires: Siglo Veintiuno Editores.

BERGER, Peter. 1969. “Religión y mantenimiento del mundo". In: . El dosel sagrado. Elementos para una sociología de la religión. Buenos Aires: Amorrortu.

BERGER, Peter \& LUCKMANN, Thomas.2006. "La sociedad como realidad subjetiva”. In: La construcción social de la realidad. Buenos Aires: Amorrortu.

BOURDIEU, Pierre. 1986. “Notas provisionales sobre la percepción social del cuerpo". In: Sociología crítica. Madrid: La Piqueta A.A.W Materiales sobre 2012. La Distinción. Criterios y bases sociales del gusto. Buenos Aires: Taurus.

BRAZ, Camilo. 2005. “O Meu Corpo é o Meu Templo: projetos corporais e normatividades no universo da body modificação em São Paulo". Humanitas (PUCCAMP). (8) 167-177. Recuperado el 20 de abril dehttps://www.sertao.ufg.br/up/16/o/ d41-cbraz.pdf

BUSSO, Mariana. 2006. “Las ferias, un lugar de encuentro, de compras, de trabajo. Un estudio de caso en la Ciudad de La Plata, Argentina". Informes de Investigación, no. 18. CEIL-PIETTE. CONICET. Recuperado el 30 de mayo de 2008 de:http:// bibliotecavirtual.clacso.org.ar/ar/libros/argentina/ceil/busso.pdf

COSTA, Zeila. 2004. Do Porão ao Estúdio: trajetórias e práticas de tatuadores e transformações no universo da tatuagem. Dissertação de Mestrado. Florianópolis: Universidade Federal de Santa Catarina.

DURKHEIM, Emile. 1968. “La Noción de Alma”. In: . Las formas elementales de la vida religiosa. Buenos Aires: Schapire.

ELIAS, Norbert. 1993. El proceso civilizatorio, Buenos Aires: Fondo Cultura Económica.

. 1998. “Ensayo teórico sobre las relaciones entre establecidos y marginados”. In: Norbert Elias (org.) La civilización de los padres y otros relatos. Bogotá: Norma.

FERREIRA, Vítor Sérgio. 2006. Marcas que Demarcam: corpo, tatuagem e body piercing em contextos juvenis. Tese de Doutorado. Lisboa: Instituto Superior de Ciências do Trabalho e da Empresa.

GIDDENS, Anthony. 1991. Modernidad e Identidad del yo. El yo y la sociedad en la época contemporánea. Barcelona: Península.

KOPYTOFF, Igor. 1991. “Biografía cultural de las cosas”. In: A. Appadurai. La vida social de las cosas. México: Grijalbo. 
LASH, Scott \& URRY, John. 1997. Economía de signos y espacios. Buenos Aires: Amorrortu.

LEITÃO, Débora Krischke. 2003. "Corpos Ilustrados: tatuagem e autonomia sobre anatomía”. In: Encontro Anual da Associação Nacional de Pós-Graduação e Pesquisa em Ciências Sociais. Anais... Caxambu: ANPOCS. Recuperado el 20 de abril de: http://portal.anpocs.org/portal/index.php?option=com_docman\&task=doc_view\&gid=4222\&ltemid=316

MONTEIRO LEITE, Elenice. 1996. "Flexibilidad y polivalencia”. In: El rescate de la calificación. Montevideo: Cinterfor.

SIMMEL, George. 2002. Sobre la individualidad y las formas sociales. Buenos Aires: Ediciones Universidad Nacional de Quilmes.

VASILACHIS DE GIALDINO, Irene. 2006. "La investigación cualitativa”. In: I. Vasilachis de Gialdino. (org.). Estrategias de Investigación Cualitativa. Buenos Aires: Gedisa. 


\section{Prácticas corporales y conversión al oficio entre tatuadores y perforadores}

\section{RESUMO}

Este artigo pretende analisar processos de consumo cultural relacionados às dinâmicas de iniciação e profissionalização de ofícios baseados em práticas corporais estéticas. Para isto ponderamos discursos e práticas de tatuadores e colocadores de Body Piercing (perfuradores) profissionais de uma cidade argentina. Com uma abordagem qualitativa utilizamos técnicas como entrevistas e observação participante. A partir de uma diferenciação geracional conseguimos reconstruir lógicas de acesso e aprendizagem do ofício entre: velhos autodidatas e jovens aprendizes. Posteriormente identificamos tatuadores que se auto-definem como artistas tradicionais, mas que no esforço de transpor o tecido à pele buscam expressar uma ruptura com o entorno social e familiar. Por último reconhecemos colocadores de Body Piercing (perfuradores) que sentem a perfuração como uma filosofia de vida, construindo discursos de diferenciação sobre si mesmos baseados em aquilo que é necessário para ser um "bom colocador de Body Piercing".

PALAVRAS-CHAVE: ofício e profissionalização; práticas corporais; metodologia qualitativa; tatuadores; colocadores de body piercing.

\section{Practices of body modification and conversion to the craft among tattoo artists and body piercers}

\section{ABSTRACT}

This paper aims to analyze processes of cultural consumption associated to practices of body modification. We focus on the dynamics of initiation and professionalization that are present within the crafts of tattooing and body piercing. To accomplish this we regarded discourses and practices of professional tattoo artists and body piercers in an Argentinian city. Using a qualitative approach we utilized techniques such as participant observation and in-depth interviews. Based on a generational differentiation we managed to reassemble logics of accessing and learning the crafts among old established self-taught individuals and young apprentices. Afterwards, we identified tattoo artists who are self-proclaimed traditional artists. Yet, these individuals were also dedicated to transposing from the canvas to the skin, seeking with this a rupture with their social and family surroundings. Lastly, we identified the case of body piercers, who feel the activity of piercing as a life philosophy. Therefore, they construct discourses of differentiation among themselves based on necessary elements that make a 'good body piercer'.

KEYWORDS: craft and professionalization; corporal practices; qualitative methodology; tattoo artists; body piercers.

Recebido em: 12/08/2015

Aprovado em:30/11/2016 